\title{
Research on Variant Design and Optimization System for Nuclear Power Valve based on CAD/CAE Integration
}

\author{
Shi Yao-jun, Dai Ye* and Wu Nan-qun \\ School of Mechanical \& Power Engineering, Harbin University of Science and \\ Technology, Heilongjiang Harbin 150080, China \\ *daiye312@163.com
}

\begin{abstract}
Aiming at the problem of long cycle and high cost in designing power valve products at present, a nuclear power valve design method for collaborative design, simulation and optimization is put forward. Through the summarized characteristics of nuclear power valve products in CAE series, the basic framework of parametric CAE analysis is constructed. Combined with parametric variant design and simplification module for valve parts, the heterogeneous information between CAD and FEA of valve models is expressed by using the universal data exchange technology. According to the integrated development framework, an integrated system, which integrates parametric design, multi-characteristic simulation, and multidisciplinary design optimization for the nuclear power valves together supporting heterogeneous model transfer, is constructed and validated through a case. Through integration of heterogeneous CAD/CAE software such as UG, ANSYS and iSIGHT, the analytical efficiency is enhanced by coupling respective and unique advantage of various software systems. This transfer is benefit for mutual coordination and information sharing that improves the automation in design and analysis on the valve products.
\end{abstract}

Keywords: collaborative design; multidisciplinary design; nuclear power valve.

\section{Introduction}

With the high-speed development of modern industry, the updating cycle of manufacturing products becomes shorter and shorter, hence, the design process in the whole life cycle of industry products gradually occupies the increasingly important position ${ }^{[1-4]}$. At present, there exist a universal phenomenon of low efficiency and high processing cost during the valve manufacture process in domestic nuclear power valve business. Although these enterprises have introduced the technologies of computer aided design (CAD) and computer aided engineering (CAE), the information transmission still rely mainly on manual transmission due to the lack of interaction and mutual support between $\mathrm{CAD}$ and $\mathrm{CAE}^{[5-7]}$. Therefore, the problem of isolated information among the CAX systems has become increasingly acute, leading to the low efficiency in the digital prototype design process, the difficulty in managing the result data and the suboptimal design performance of valve products ${ }^{[8]}$. Obviously, these shortcom-ings greatly affect the development speed and design quality of valve products, so it is an urgent need to establish a practical CAX integrated design system.

In recent years, many experts and scholars at home and abroad have completed a lot of research and development works on product design platform. In order to achieve the integration analysis function of product feature, Seungho constructs an integrated analysis system by using JADE (Java Agent DEvelopment), and takes the automotive suspension design as an example to verify the effectiveness of the platform ${ }^{[9]}$. Naranje develops a 
computer aided integrated design system of deep drawing dies, which consists of modeling module, analysis module and assembly simulation module, and these modules are supported by knowledge base of the die component design ${ }^{[10]}$. Aiming at the structural features of some frame parts in marine diesel engine manufacturing enterprise, Zhang studies the key technologies for feature information extraction, feature matching, feature reconfiguration, feature process inference and feature tool path, and develops an integration system of marine diesel engine frame parts and validates it using a case ${ }^{[11]}$. In order to solve the shortcomings of existing innovation of computer-aided design software with respect to problem analysis tools, innovative solution strategy and knowledge base, Chen puts forward a model of computer-aided innovation design platform and verifies its integrity and feasibility ${ }^{[12]}$. The research methods mentioned above establish the integrated simulation platform through the integration between $\mathrm{CAD}$ and $\mathrm{CAE}$, and use the database technology to complete the transmission and storage of product information, thus to realize the integrated development of the products from design to the simulation optimization. However, most of these platforms are geared to the needs of certain specific product development and aren't suitable for the nuclear power valve design process.

\section{Program Analysis of System Integration}

\subsection{Function Analysis of the Integrated System}

According to the characteristics of the digital design process and requirements for the power station valve products, the integrated system functions for collaborative design, simulation and optimization mainly reflect in the following four main aspects, as shown in Figure 1:

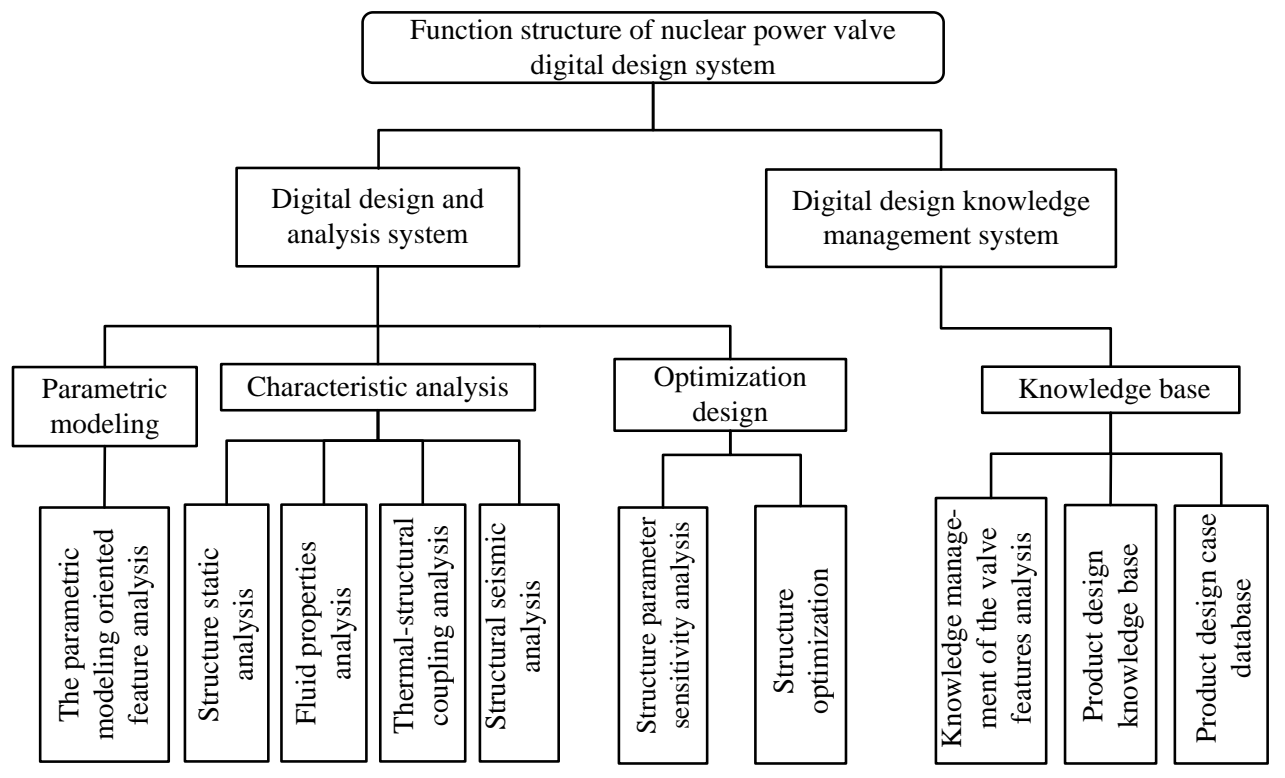

Figure 1. Digital Design System Function Structure of Nuclear Power Valve

(1) nuclear power valve structural parametric design, which is the guarantee to realize the fast analysis and structure optimization technology; (2) nuclear power valve multi-physical field analysis, which includes the valve body structure analysis, the flow 
properties analysis, the thermal - stress coupling analysis and the seismic analysis; (3) nuclear power valve structure optimization, the main task of which is to optimize the nuclear power valve structure according to the results of the multi-physical field analysis; (4) digital design knowledge management, which can guide the analyst to carry on the analysis more conveniently so as to improve the model quality and the characteristic analysis efficiency.

\subsection{System Workflow Design}

The whole system adopts initial design data of the valve product as the origin, including parametric modeling and assembly, multi-physics field analysis, structural optimization design of the nuclear power valve products, as illustrated in Figure 2. The main steps of the integration system are shown as following:

(1) Parametric modeling and assembly of the valve products: the initial design data can be determined according to customer's requirements, and parametric CAD models of the valve parts would be established and assembled together, which will be used in CAE environment;

(2)Valve multi-physics field analysis: the analysis model will be imported into multiphysical field simulation system through a neutral file. Aiming at the different analysis demands, the system could assist to complete the performance simulation preprocessing work such as meshing, adding constraints, and so on. After completing the performance analysis work under the different working conditions of the nuclear power valve, the lack of the structure design can be pointed out that will provide beneficial reference for the optimization design;

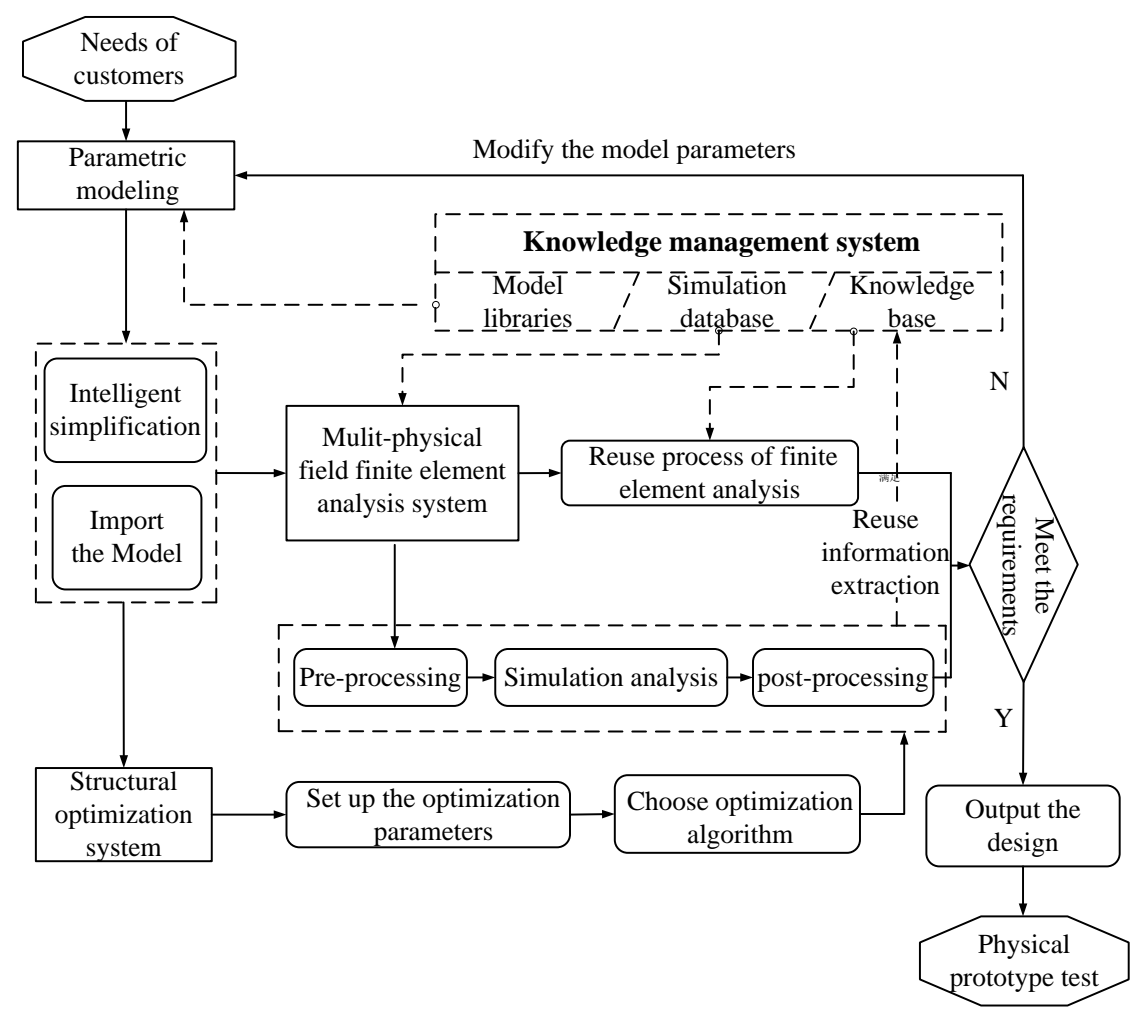

Figure 2. Platform Flow Chart of Nuclear Power Valve Digital Design 
(3) Valve structure optimization design: after completing the valve performance analysis, the optimized analysis should be carried out by inputting relevant structure parameters and optimization algorithm. Finally, the optimized design scheme of the valve structure will provide a reference for the physical prototype manufacturing information;

(4) Knowledge management system: as an information system of the whole design process, it provides relevant design parameters, such as the enterprise test data, the standards and specifications for the valve design, the fluid properties and parameters, and so on.

\section{Key Technologies of System Integration}

\subsection{System Structure of the Digital Design}

According to the specific structure characteristics and working requirements of the nuclear power valve products, the numerical analysis and evaluation for the nuclear power valve can be divided into four aspects: the structure strength analysis, the thermal change analysis, the flow ability in the cavity of the valve body and the valve seismic performance. The digital design system structure is constructed to satisfy the requirements of the performance simulation analysis of the nuclear power valve products. Therefore, the system structure are established according to the level structure, it mainly consists of three major components, including the supporting layer, function layer and application layer. In the function layer, the parametric design logical processing is based on UG, the comprehensive characteristic analysis logical processing based on ANSYS; the structure optimization design logical processing based on the ISIGHT, and these logical modules are integrated together to the tool software.

\subsection{Parametric Model based on Features}

The geometry topology relation usually changes little for same type valves, hence, the method that modifying model template by calling DLL is adopted to generate parametric model of valve parts automatically. This method modifies model fast and stable, and is convenient for designers who are unfamiliar with UG operating. And the SQLsever database is integrated into the system in order to manage and call the parameter data of each part model systematically. Its organizational structure is shown in Figure 3.

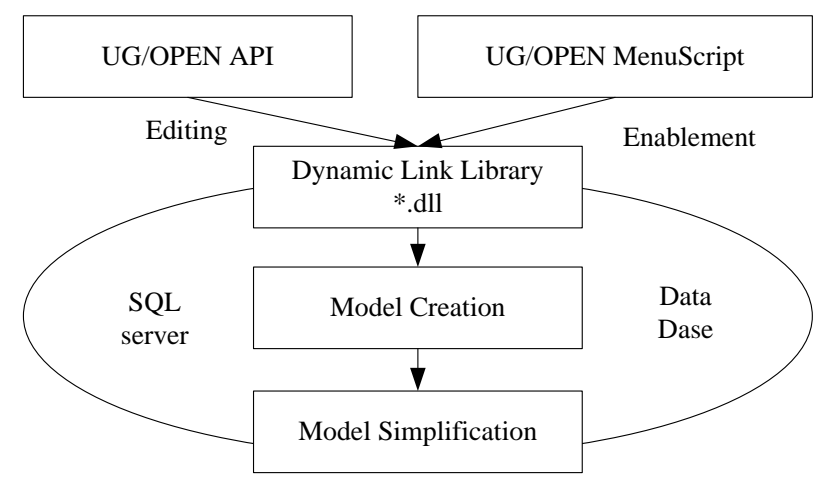

Figure 3. Platform Structure of Valve Parametric Modeling 
The modeling method is based on the features in the process of building valve's parametric models, which helps to express more specific features of the model and be convenient for operator to programme. Parameter function is realized by integrated UG software, and the process of integration is finished by second development interface of UG/OPEN. When using parametric models for typical parts of the nuclear valve, the building of each parameter is based on feature size. Parameter's name is divided into four levels according to the need of data management and programming, the specific writing format is: serial number_parts number_feature number_ name abbreviation. For example, V2082_012_01_Hol4_D is the name of valve inlet, it can be seen that this parameter belongs to the fourth pore characteristics diameter of number one part of V2082_012 series.

If a certain parameter is marked out by numerical method on the drawing, and it doesn't rely on other characteristics for localization, then this parameter can be defined as independent parameter and endowed with fixed value in the UG Expressions editor. It changes model size by using UF_MODL_edit_exp function and renewals expression by UF_MODL_update function. For the parameters that have fixed algorithm, it requires to provide round processing according to the design principle and practical processing requirements when compiling expression. For example, the empirical formula of stepped shaft's blade shoulder diameter is 1.2 times of axis diameter, hence the result of expression may not be an integer, and it needs to be rounded according to the design requirement. Considering the strength, "ceil()" function should be added to the expression for rounding up, and the other parameters which are independent to strength are rounded down by using "floor()" function according to non-interference requirement.

\subsection{Simplification Module of Valve Parts}

The results of the experiments and simulations indicate that some small features on the valve body does not affect the results of the structure analysis, but the presence of these features would affect the mesh division and cause the mesh too close around the holes, which will affect the efficiency and accuracy of FE analysis. Thus, the simplification module of valve typical parts is developed to improve the efficiency of valve analysis. When the simplification module handles the valve body model, the size control parameters and the simplification sensitivity should be inputted, and the small features can be deleted according to presetting condition. This proposed method can overcome the shortcomings of heavy workload and low efficiency in model simplification process, and has a high effectiveness at building finite element analysis models.
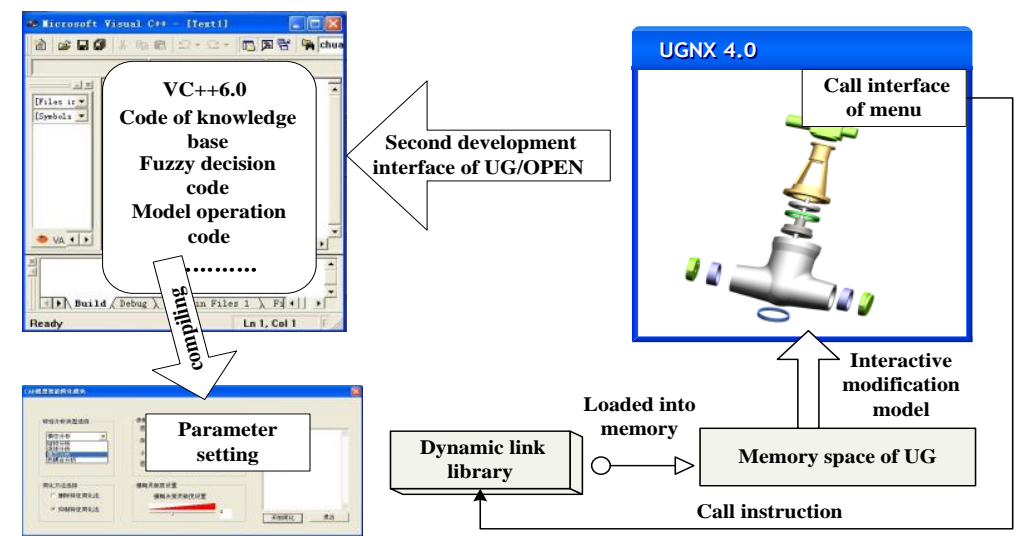

Figure 4. Model Simplification Operator Interfaces 
The simplification module uses UGNX4.0 for CAD modeling platform, and takes the second development interface of UG/OPEN to implement the simplification process in the development environment of $\mathrm{VC}++6.0$. As shown in Fig. 4, the development process of this simplification module is as follows:

STEP1: Use the function of UF_PART_ask_display_part () to obtain the identification of current parts model; and start the while loop by the function of UF_OBJ_cycle_objs_in_part() to traverse the entire model features tree;

STEP2: Determine the type of the feature by the function of UF_MODL_ask_feat_type(), and then set the evaluation function by the program(such as Value_Boss() is the evaluation function of a cone);

STEP3: Write the model features into the linked list according to the evaluation results, which then are displayed in the interface as a technical reference for the user;

STEP4: According to the selection of user, call the function of UF_OBJ_delete_ object () or UF_MODL_suppress_feature characteristics ()to remove or restrain the features ;

STEP5: Save the model with neutral format, and exit the module.

\section{Implementation and Application of the System}

\subsection{System Implementation}

The nuclear power valve integrated system for collaborative design, simulation and optimization is developed based on VC and SQL Server database system. Through providing a unified the data access interface, the system make each simulation system be flexible to define and publish the data sets so as to realize the information integration in the multidisciplinary simulation process.

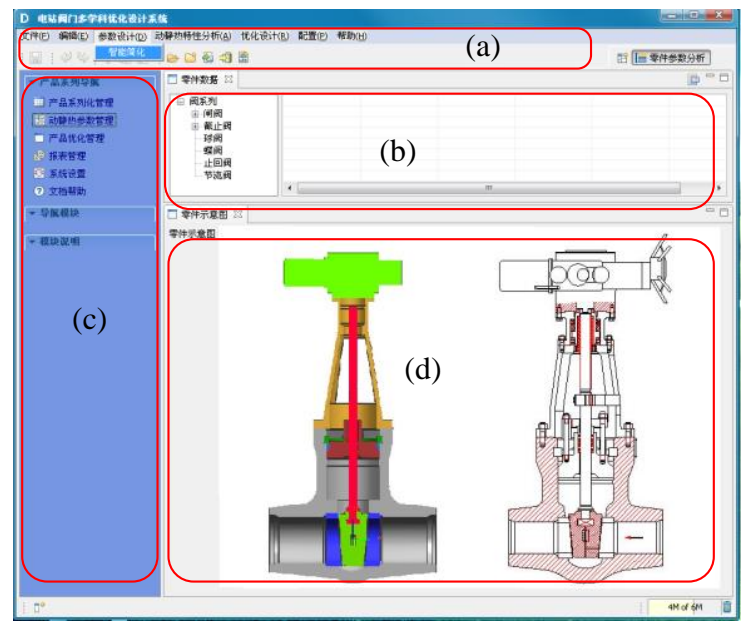

Figure 5. Main Interface of the Integration System

The main interface of the integrated system for valve products is shown in Fig.5, which is divided into four functional areas, viz. navigation area (a), which contains task bar button access into the three design platform; parts list area (b), the system will list the main parameters of the components after the choice of valve type or model number; system management list area (c), which is used to save and manage the results from the three design systems; parts diagram area (d), which provides the diagram of the parts for the operator, including 3D modeling graphics and engineering drawing. 


\subsection{System Application}

The supercritical electric gate valve (DN230-CL2680) is selected as an example to study, the main performance parameters of the electric gate valve as shown in Tab.1. In order to comprehensively analyze the specific impact of the nuclear power valve under various influence factors, multi-physics field analysis of the whole valve, including structural static analysis, thermal-mechanical coupling analysis, flow field analysis, Seismic analysis, should be completed to predict the valve performance under different work conditions.

Table 1. Main Performance Parameter of Gate Valve DN230-CL2680

\begin{tabular}{cccccccc}
\hline $\begin{array}{c}\text { Pressure } \\
\text { Class }\end{array}$ & $\begin{array}{c}\text { Design } \\
\text { Pressure } \\
/ \mathrm{MPa}\end{array}$ & $\begin{array}{c}\text { Design } \\
\text { Temperature } \\
{ }^{\circ} \mathrm{C}\end{array}$ & $\begin{array}{c}\text { Working } \\
\text { Pressure } \\
/ \mathrm{MPa}\end{array}$ & $\begin{array}{c}\text { Working } \\
\text { Temperature } \\
/{ }^{\circ} \mathrm{C}\end{array}$ & $\begin{array}{c}\text { safety } \\
\text { level }\end{array}$ & $\begin{array}{c}\text { anti- } \\
\text { seismic } \\
\text { leve }\end{array}$ & $\begin{array}{c}\text { Connection } \\
\text { type }\end{array}$ \\
\hline $\begin{array}{c}\text { Class } \\
2680\end{array}$ & 30.1 & 441 & 27.5 & 365 & First & First & BW \\
\hline
\end{tabular}

The whole process is shown as Figure 6, first of all, designers should choose the analysis object in the part data lists and enter the model selection interface, and then can choose the appropriate model as a design template. After inputting all parameters of valve analysis, designers can get into the characteristics analysis platform by finite element analysis. Each analysis module manages the data of the CAE model, simulation analysis and post-processing graphic. Later, the analysis results will be displayed in the form of a chart. Finally, the optimum module will optimize the structure of the valve.

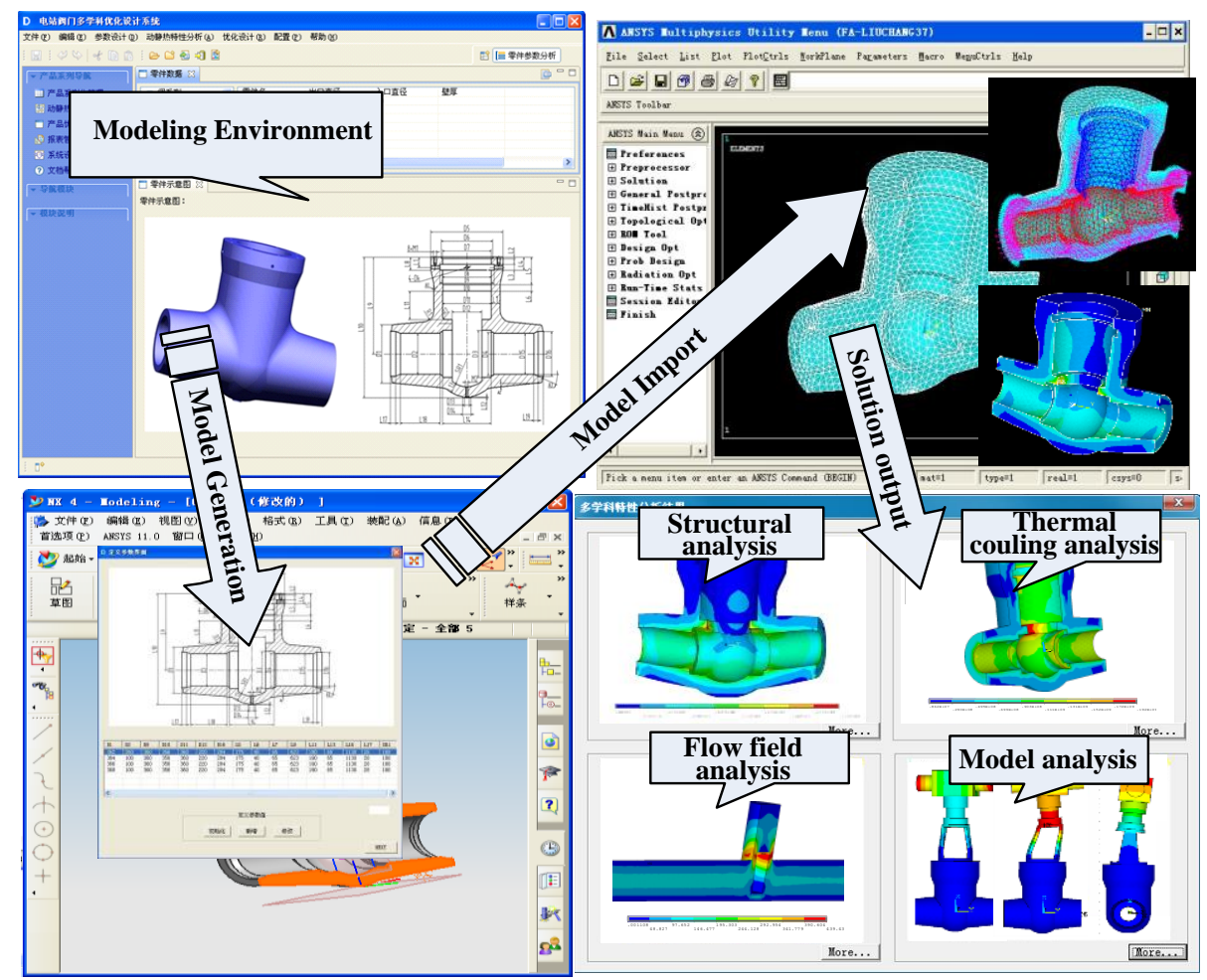

Figure 6. Main Process of the Integrated Design System 


\section{Conclusion}

In order to verify the validity of the integrated design system of nuclear power valves, multi-physics analyses are completed on the supercritical electric gate valve (DN230-CL2680). In view of the demands in multidisciplinary design optimization (MDO) of valve products, the integrated design system for valves is constructed, which integrates the design, simulation and optimization of nuclear valves. On this basis of integrated design system, the multi-physics analyses of the electric gate valve of DN230-CL2680 are carried out, which takes account of mechanical analysis, fluid mechanics analysis, thermal-mechanical coupled analysis and seismic analysis. The validity of the integrated design system above is verified, so the simulation of the valve can effectively improve the accuracy and efficiency of designing the valve products.

\section{Acknowledgements}

The research is supported by Heilongjiang Province Education Bureau Project (Grant No. 12541146).

\section{References}

[1] K. H. Chang and S. H. Joo, "Design parameterization and tool integration for CAD-based mechanism optimization[J]", Advances in Engineering Software, vol. 37, no. 12, (2006), pp. 779-796.

[2] J. Bai, S. Gao, W. Tang, et al., "Design reuse oriented partial retrieval of CAD models [J]", Computer-Aided Design, vol. 42, no. 12, (2010), pp. 1069-1084.

[3] L. Mingzhu, D. Ye and Z. Guoyin, "Research on CAD/CAE/MDO integrated system for nuclear valve products [J]", Journal of Harbin Institute of Technology (New Series), vol. 19, no. 4, (2012), pp. 99-103.

[4] C. Antonio, K. G. Satyankra, D. Abhijit, et al., "Machining feature-based similarity assessment algorithms for prismatic machined parts[J]", Computer-Aided Design, vol. 38, no. 9, (2006), pp. 954-974.

[5] D. Ye, L. Yinan, G. D. Todorov, et al., "Structural optimization of high pressure bypass-valve body for 600MW supercritical units[J]", Journal of Harbin Institute of Technology (New Series), vol. 17, no. 1, (2010), pp. 168-170.

[6] Y. L. Perry, "Using steady flow force for unstable valve design: modeling and experiments [J]", Department of Mechanical Engineering, vol. 27, (2005), pp. 39-42.

[7] R. Amirante, G. D. Vescovo and A. L. Ippolis, "A flow forces analysis of an open center hydraulic directional control valve sliding spool[J]”, Energy Convers Manage, vol. 47, (2006), pp. 114 -131.

[8] D. Ye, L. Yinan, He. Xibin, et al., "Design and implementation of MDO integrated system for valve products [J]”, Advanced Materials Research, vol. 308, (2011), pp. 1273-1276.

[9] H. Seungho, J. Lee, T. Lee, et al., "Parametric study using an integrated design system to assessfatigue durability of control arms in automobile suspension module [C]", The 11th International Conference, vol. 52, no. 36, (2007), pp. 144-154.

[10] V. Naranje and S. Kumar, "An intelligent CAD system for automatic modelling of deep drawing die [J]", International Journal of Computer Applications in Technology, vol. 48, no. 4, (2013), pp. 330-344.

[11] S. W. Zhang, Y. L. Ding, G. C. Wang, et al., "CAD/ CAPP/ CAM integration system for frame parts of marine diesel [J]", Computer Integrated Manufacturing Systems, vol. 17, no. 4, (2011), pp. 760-766.

[12] L. Chen, Y. Li, W. Q. Li, et al., "Development of a computer-aided product innovation and design systemCAIP[J]”, Computer Integrated Manufacturing Systems, vol. 19, no. 2, (2013), pp. 319-329. 